\title{
Resolution of Racemic Guaifenesin Applying a Coupled Preferential Crystallization-Selective Dissolution Process: Rational Process Development
}

\author{
Erik Temmel, ${ }^{\dagger}$ Matthias J. Eicke, ${ }^{\dagger}$ Francesca Cascella, $^{\ddagger}$ Andreas Seidel-Morgenstern, ${ }^{\dagger \dagger}$ \\ and Heike Lorenz ${ }^{*} \dagger$ \\ ${ }^{\dagger}$ Max Planck Institute for Dynamics of Complex Technical Systems, Sandtorstraße 1, 39106 Magdeburg, Germany \\ ${ }^{\ddagger}$ Otto von Guericke University Magdeburg, Chair of Chemical Process Engineering, 39106 Magdeburg, Germany
}

\section{Supporting Information}

\begin{abstract}
Preferential crystallization is a cost efficient method to provide pure enantiomers from a racemic mixture of a conglomerate forming system. Exploiting small amounts of pure crystals of both enantiomers, several batch or continuous processes were developed, capable of providing both species. However, an intermediate production step has to be used when pure enantiomers are not available. In such cases, partially selective synthesis, chromatography, or crystallization processes utilizing chiral auxiliaries have to be used to provide the initial seed material. Recently, it was shown that a coupled Preferential Crystallization-selective Dissolution process (CPCD) in two coupled crystallizers can be applied if at least one pure enantiomer is available to produce both antipodes within one batch. The corresponding process is carried out in one reactor (crystallization tank) by seeding a racemic

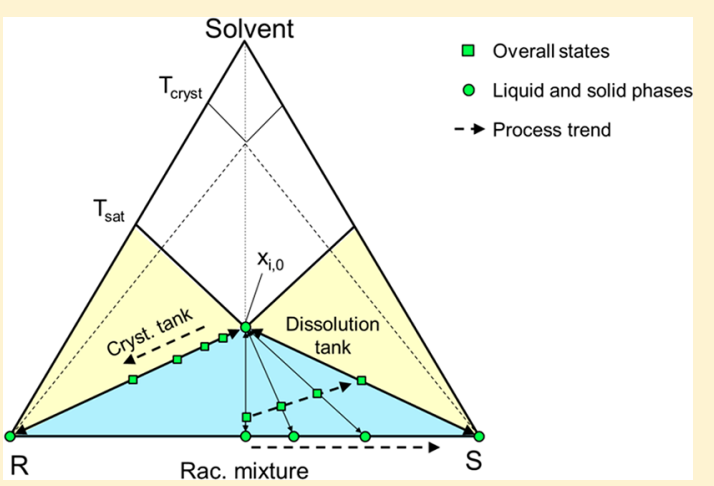
supersaturated solution with the available enantiomer at a certain temperature. The second reactor (dissolution tank) contains a saturated racemic suspension at a higher temperature. Both reactors are coupled via the fluid phase, allowing for a selective dissolution of the preferentially crystallizing enantiomer from the solid racemic feed provided in the dissolution vessel. The dissolution and crystallization processes continue until the solid racemic material is completely resolved and becomes enantiopure. At this point, both enantiomers can be harvested in their pure crystalline form. For a specific pharmaceutically relevant case study, a rational process design and the applied empirical optimization procedure will be described. The achieved productivities after optimization show the great potential of this approach also for industrial applications. Also, a strategy to control this process based on inline turbidity measurement will be presented.
\end{abstract}

\section{INTRODUCTION}

Enantiomers are molecules that are mirror images of each other (optical isomers) with the same molecular structure and identical physicochemical properties. The stereoisomerism of two enantiomers can cause different effects if they are applied to biological systems due to chirality of enzymes, hormones, etc. Nowadays, there is evidence that commonly only one enantiomer induces the desired effect while the other is inactive or even harmful. ${ }^{1,2}$ Thus, if chiral components are utilized as a racemic mixture (50/50 ratio of both enantiomers), half of the applied material can be toxic, cause stress for the biological system, or is wasted leading to environmental pollution.

The development of processes to produce pure enantiomers is therefore a prerequisite for economically and ecologically effective applications of chiral substances in, e.g., the pharmaceutical, food, and agrochemical industries. Even though a direct synthesis of one single stereoisomer is preferred, it is often expensive or impossible. ${ }^{3}$ Alternatively, the substances can be conventionally produced as racemic mixtures and separated subsequently with appropriate techniques exploiting chromatography, membranes, or, like in the present study, preferential crystallization. ${ }^{3-5}$

In order to make use of preferential crystallization, beside the basic requirement that the racemate to be separated forms a conglomerate, a suitable amount of homochiral crystals is initially necessary to gain reasonable yield and productivity. The availability of pure enantiomers can be a bottleneck. In this study, only a very limited amount of one enantiomer of guaifenesin was available from a complex asymmetric synthesis. ${ }^{6}$ Additionally, not much was known regarding crystallization kinetics and also solubility data were insufficient.

An innovative approach is studied in this article to produce material for further investigations that would circumvent possible loss or contamination of the valuable starting material, which can be a result of unknown thermodynamic or kinetic

Received: November 5, 2018

Revised: February 25, 2019

Published: May 1, 2019 
a)

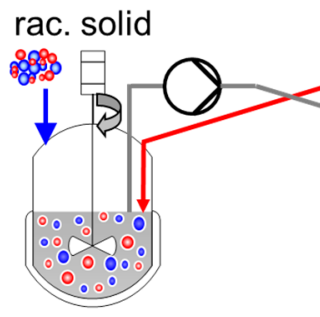

$\left(T_{\text {sat }}, x_{i, 0}\right)$

Dissolution

tank
Seeding $(R)$

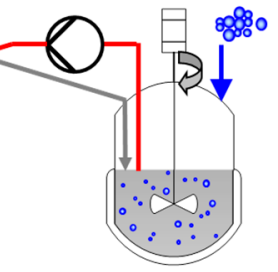

$\left(T_{\text {cryst }}, \mathrm{X}_{\mathrm{i}, 0}\right)$

Crystallization

tank

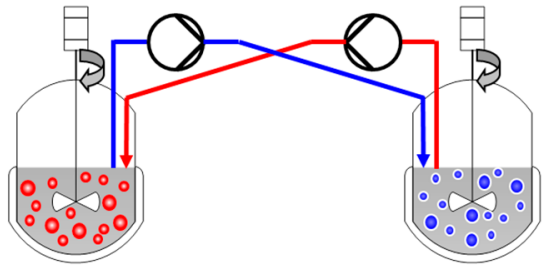

$\left(T_{\text {sat }}, x_{i, 0}\right)$

Dissolution

tank
$\left(T_{\text {cryst }}, X_{i, 0}\right)$

Crystallization

tank
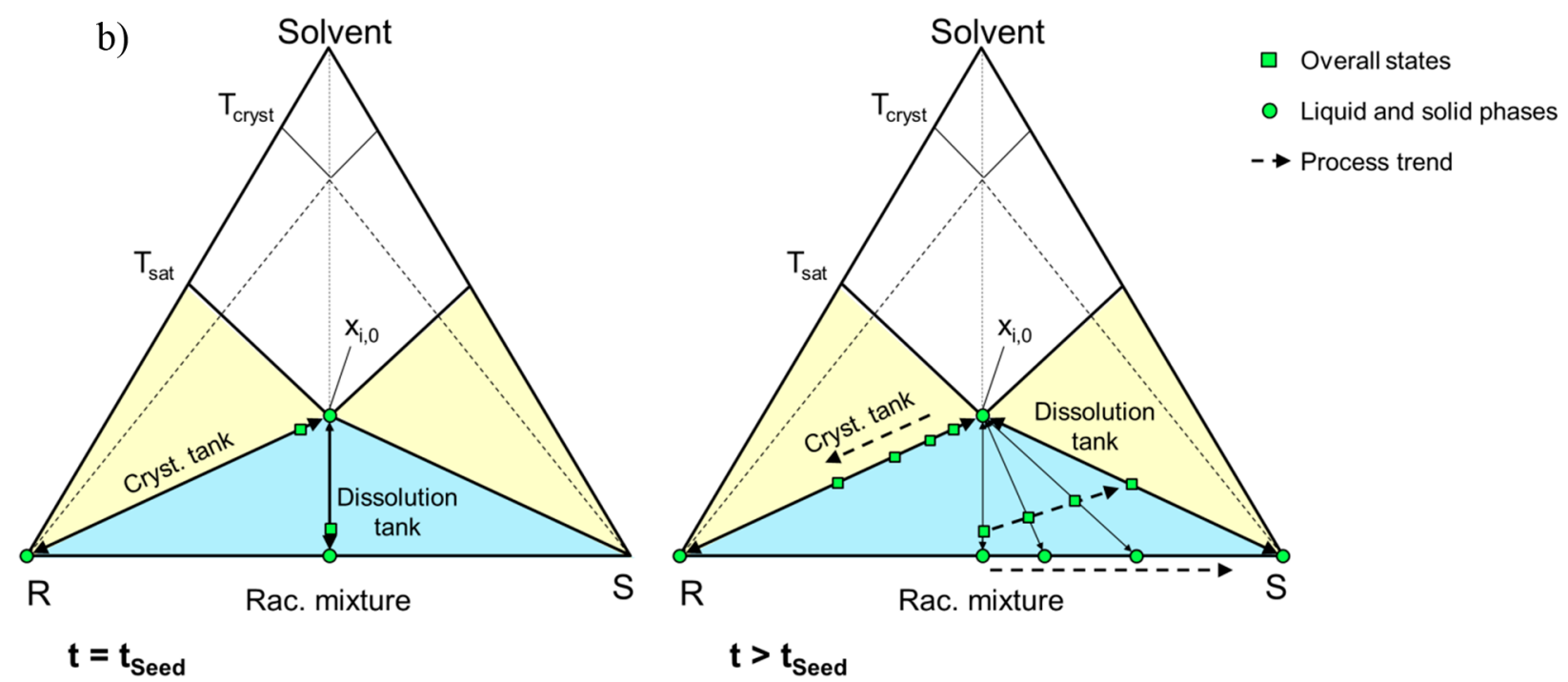

Figure 1. (a) Schematic representation of the initial situation after seeding and the separation result of the Coupled Preferential CrystallizationDissolution process (CPCD). (b) Representation of the composition evolution by means of a ternary phase diagram depicting the state changes in the dissolution and crystallization tank for the ideal separation process. Right after seeding $\left(t=t_{\text {seed }}\right.$, left figure $)$ only a small amount of the solid $(R)$-enantiomer is present in the crystallization tank while the dissolution tank contains a large amount of crystalline racemic mixture. Afterward $(t$ $>t_{\text {seed }}$, right figure), the mass of pure, solid $(R)$-enantiomer increases due to the preferential crystallization and the overall state in this vessel moves toward the pure phase corner. Selective dissolution of the $(R)$-enantiomer in the dissolution tank yields a decrease of the overall crystal fraction in suspension and an enrichment of the $(S)$-enantiomer in the solid phase, which moves the solid state composition in this tank to the pure $(S)$-phase corner.

information. The process is based on a preferential crystallization of only one pure enantiomer combined with its selective dissolution from a solid mixture of racemic composition utilizing two different tanks, which are coupled via the liquid phase. ${ }^{7,8}$ Below we will designate this "Coupled Preferential Crystallization with selective Dissolution Process" as CPCD. It is ideally suited to safely amplify a small amount of starting material while additionally gaining access to the counter-enantiomer in the form of crystals.

1.1. Process Principle. The process is carried out in two tanks that are initially filled with saturated solutions of racemic composition $\left(x_{i, 0}\right.$, Figure 1$)$ at one specific temperature $\left(T_{\text {sat }}\right.$ Figure 1). Solid racemic feed is provided in excess in the first tank, which remains at saturation temperature $T_{\text {sat }}$ and serves as the subsequent dissolution stage (dissolution tank in Figure 1 a). The second tank (crystallization tank in Figure 1a) is cooled to a specific temperature $T_{\text {cryst }}$ where an appropriate driving force for crystallization can be expected $\left(\Delta T=T_{\text {sat }}-\right.$ $T_{\text {cryst }}$ ), but primary nucleation does not occur. Afterward, the separation is initiated in this crystallization tank by adding a small amount of pure crystals of one enantiomer (here $(R)$ ).
These crystals will start to grow due to the present supersaturation and, thereby, selectively remove the seeded enantiomer from the liquid phase indicated by its decreasing concentration in the mother liquor. The $(R)$-depleted liquid phase is continuously transported to the dissolution tank that is still kept at the higher saturation temperature $T_{\text {sat }}$. As a result, the seeded $(R)$-enantiomer becomes undersaturated in the dissolution vessel, which leads to its selective dissolution from the present racemic solid phase (changing solid phase composition in the dissolution tank, Figure $1 \mathrm{~b}$ ), thus restoring the saturation concentration at $T_{\text {sat }}$. By coupling both reactors via their liquid phases, the mother liquors in both vessels remain at racemic composition if the exchange and the dissolution processes are fast enough to counterbalance the selective concentration decrease by the preferential crystallization.

The situation right after seeding is reflected in the left ternary phase diagram in Figure $1 \mathrm{~b}$. Initially, only a small, enantiopure solid fraction is present in a supersaturated racemic liquid phase at $T_{\text {cryst }}$ while, in the dissolution tank, a large amount of racemic solid phase is in equilibrium with a 
racemic liquid phase saturated at $T_{\text {sat }}$. In the ideal case, the seeded enantiomer is preferentially crystallized and primary nucleation of the antipode is efficiently prevented, which results only in an increasing solid mass in the crystallization tank while the liquid phase composition stays racemic (composition $x_{i, 0}$, Figure $1 \mathrm{~b}$ ). This is depicted in the right ternary phase diagram in Figure $1 b$, where the liquid and solid composition in the crystallization tank is constant, while the overall state (reflecting the ratio between the masses of the liquid and solid phase) moves toward the corner of the pure $(R)$-enantiomer. By contrast, the solid mass decreases in the other tank due to the selective dissolution that changes simultaneously the enantiomeric ratio in the present crystal phase. Hence, the solid state (right phase diagram in Figure $1 b)$ of the dissolution tank moves toward the pure $(S)$ enantiomer and the changing overall state reflects the loss of solid content. Also, in this tank, the liquid phase is of racemic composition if the selective dissolution counterbalances the preferential crystallization and the exchange rate between both vessels is sufficiently high.

The process ends after the seeded enantiomer is completely dissolved from the racemic excess material in the dissolution tank (Figure 1a). If the process is interrupted at exactly this time, only the seeded enantiomer is present as solid in the crystallizer, while, in the dissolution tank, only the unseeded enantiomer as solid is left. Further crystallization leads to an enrichment of the unseeded enantiomer in the crystallization tank and, similarly, to conventional preferential crystallization, which increases the risk of primary nucleation.

1.2. Separation Problem. The API guaifenesin (3-(2methoxyphenoxy)-propane-1,2-diol (Figure 2) was chosen as

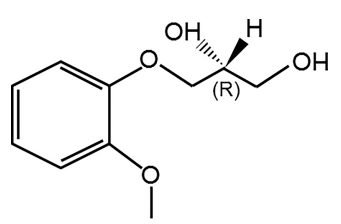

Figure 2. Chemical structure of $(R)$-guaifenesin. model substance to demonstrate the strength of this process strategy given such a difficult initial situation (small amount of enantiopure seed material of only one antipode, little knowledge about the substance system). Racemic guaifenesin is widely applied as an antitussive with an expectorant and decongestant effect. Nevertheless, the substance is also a valuable precursor for other pharmaceuticals like muscle relaxants $\left(\right.$ methocarbamol $\left.^{9}\right)$, tranquilizers (mephenoxalone ${ }^{9}$ ), and $\beta$-blockers (levomoprolol ${ }^{6}$ ) that are partly used in enantiopure form in therapy. Some of the latter substances form "true" racemates (racemic compounds) and are therefore not suitable for a direct and effective resolution via preferential crystallization without previous treatment. Guaifenesin, on the contrary, forms a stable conglomerate in water, and a procedure to access the pure enantiomers batchwise from enriched and racemic solutions was already reported. ${ }^{6,10}$

\section{EXPERIMENTAL SECTION}

2.1. Substance System. Racemic guaifenesin was purchased from TCI Deutschland $\mathrm{GmbH}$ (purity > 98\%) and used without further treatment. Water as solvent for the separation experiments and the washing procedure was deionized with a milli-Q system (Merck Millipore, Milli-Q Advantage).

Pure $(R)$-guaifenesin was provided by the Arbuzov Institute of Organic and Physical Chemistry (Prof. Alexander A. Bredikhin, Russian Academy of Sciences, Kazan) with a total mass of $0.8 \mathrm{~g}$.

Guaifenesin dissolved in water is known to form a conglomerate ${ }^{10}$ without occurrence of solvates or other polymorphs over the temperature and composition range of interest. It crystallizes in an orthorhombic crystal lattice ${ }^{11}$ (space group $P 2{ }_{1} 2_{1} 2_{1}$ ) with a needlelike habit of the macroscopic particles (Figure 3a).

Solubility data of the racemic mixture and the $(R)$-enantiomer measured with a polythermal method ${ }^{10}$ and utilized for the design of the first experiment are shown in Figure $3 b$. The solubilities of both the racemic mixture and the pure enantiomer show a strong nonideal and nonlinear behavior, rather two lines with a bend in between, indicating a potential phase change. However, during this study (ref 10 ), only the known polymorph was found and hydrate formation was not detected. Though, the occurrence of a second liquid phase, i.e., a stable or metastable liquid-liquid demixing, was indicated. ${ }^{10}$ Other physical properties of the substance system are listed in Table 1.

- Rac. guaifenesin (polythermal method) [10]

a Rac. guaifenesin (isothermal method) [10]

a (R)-guaifenesin (polythermal method) [10]

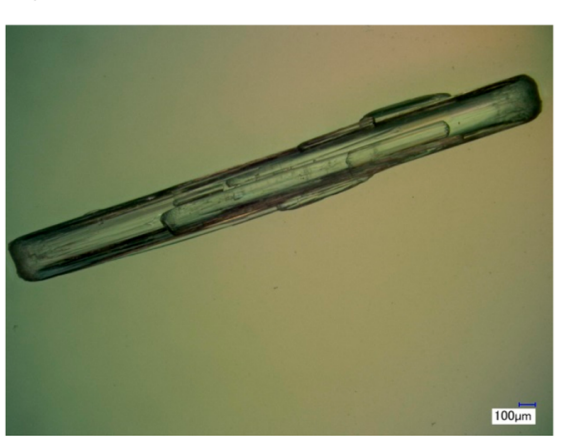

b)

a)

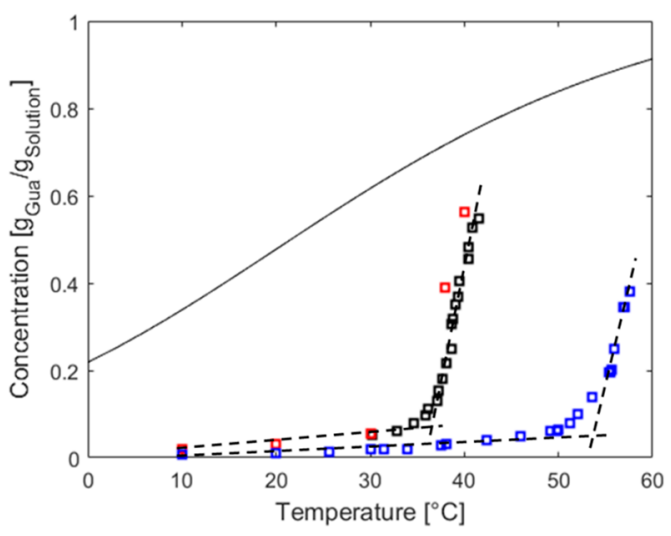

Figure 3. (a) Microscopic image of pure (R)-guaifenesin after recrystallization. (b) Solubility of racemic (black and red squares) and ( $R$ )guaifenesin (blue squares) taken from ref 10 in water in comparison to the ideal solubility curve calculated according to Schröder-van Laar (black solid line). Dashed lines are just guides for the eye. 
Table 1. Physical Properties of Solid Racemic Guaifenesin and a Saturated Solution at $36^{\circ} \mathrm{C}$

\begin{tabular}{|c|c|c|}
\hline \multicolumn{3}{|c|}{ solid state properties (safety data sheet ${ }^{12}$ ) } \\
\hline molar mass & 198.22 & $\mathrm{~g} / \mathrm{mol}$ \\
\hline melting point & $78-83$ & ${ }^{\circ} \mathrm{C}$ \\
\hline boiling point & 215 & ${ }^{\circ} \mathrm{C}$ \\
\hline density & 1.2 & $\mathrm{~g} / \mathrm{cm}^{3}$ \\
\hline \multicolumn{3}{|c|}{ solid state properties from own measurements } \\
\hline melting point ${ }^{a}$ & 78.6 & ${ }^{\circ} \mathrm{C}$ \\
\hline enthalpy of fusion ${ }^{a}$ & 191.4 & $\mathrm{~J} / \mathrm{g}$ \\
\hline density $^{b}$ & 1.25 & $\mathrm{~g} / \mathrm{cm}^{3}$ \\
\hline \multicolumn{3}{|c|}{ properties of a sat. solution $\left(36{ }^{\circ} \mathrm{C}, 0.198 \mathrm{~g}_{\text {gua }} / \mathrm{g}_{\text {solution }}\right)$} \\
\hline density $^{c}$ & 1.03 & $\mathrm{~g} / \mathrm{cm}^{3}$ \\
\hline viscosity & 1.21 & $\mathrm{mPa} \cdot \mathrm{s}$ \\
\hline $\mathrm{pH}$ & 8.1 & - \\
\hline
\end{tabular}

${ }^{a}$ Measured via DSC (Setaram, DSC 131). ${ }^{b}$ Measured via volume expansion of a saturated solution by addition of a defined solid mass. ${ }^{c}$ Measured via densitometry (Mettler Toledo, DE40).

2.2. Basic Thermodynamic and Kinetic Investigations. Isothermal solubility measurements and small-scale cooling crystallizations of the racemic guaifenesin were carried out to complement the data from ref 10 and to verify the evidence of a potentially occurring second liquid phase.

$15 \mathrm{~mL}$ portions of a suspension of water and racemic guaifenesin were prepared for each temperature to investigate the solubility behavior. Each vial was sealed and equipped with a magnetic stirrer for mixing. All samples were then placed in thermostated doublewalled glass tanks and were initially heated to dissolve the solid phase completely. The samples were recrystallized subsequently by cooling to the temperature of interest and left for equilibration $(t>48 \mathrm{~h})$. Afterward, $2-3 \mathrm{~mL}$ of liquid phase was withdrawn, weighed, and placed in a vacuum oven for drying. The mass of the residual solid phase eventually yielded the solubility at the respective temperature. At least three different samples were prepared for each temperature step (temperature range $5-50{ }^{\circ} \mathrm{C}$ ), where a part of the clear liquid phase of each sample was collected at least two times for concentration analysis.

To investigate the metastable zone width and additionally the occurring phases, a multireactor system was utilized (Crystalline, Technobis Crystallization Systems). This measurement system enables polythermal experiments with overhead stirring and observation of the samples by camera and light scattering. Samples were prepared in $6 \mathrm{~mL}$ glass vials for seven different concentrations corresponding to saturation temperatures between 28 and $43{ }^{\circ} \mathrm{C}$. The vials were subsequently cooled linearly until the lowest possible temperature was reached $\left(-20{ }^{\circ} \mathrm{C}\right)$ or when a turbidity drop indicated the occurrence of a solid phase. Altogether, five different cooling rates were investigated ranging from -0.5 to $-80 \mathrm{~K} / \mathrm{h}$. Image data around the identified nucleation points were inspected afterward with respect to the occurring phase and to possibly correct the turbidity measurement. All prepared concentrations were measured 5-20 times for each cooling rate (except for $-0.5 \mathrm{~K} / \mathrm{h}$, where only 2 measurements were possible).

2.3. Experimental Setup for Resolution Trials. All separation experiments applying the CPCD process were carried out in two 450 $\mathrm{mL}$ double-walled crystallizers (Figure 4) equipped with Teflon coated propeller type stirrers (stirrer motor: Heidolph, RZR 2102 control) and thermostats for temperature control (Lauda, Proline RP $845)$. Both tanks are connected to a bypass for online analyses, which consist of a polarimeter (IBZ Messtechnik, POLARmonitor) and a measurement of the total concentration. Two different properties, density (Mettler Toledo, DE40) and UV absorption (KNAUER, UVDetector K-2501), were investigated for concentration analysis since no appropriate process online measurement for the considered concentration range of guaifenesin is reported up to now. Both loops are fed with a crystal-free liquid phase by peristaltic pumps (Heidolph, PD 5201 SP Quick) at a rate of $3.5 \mathrm{~mL} / \mathrm{min}$.

The mother liquor exchange during the actual experiments was carried out by gear pumps (Tuthill, D-Series, $\dot{V}=0-30 \mathrm{~mL} / \mathrm{min}$ ) connected to mass-flow controllers (Bronkhorst Maettig GmbH, mini Cori-Flow M14), which enable a precise volume control in both tanks as well as a measure of temperature and density of the present liquid phase. All tubes are heated slightly above saturation temperature via thermostats (Lauda, Ecoline RE 104, Julabo, FS 18) to prevent

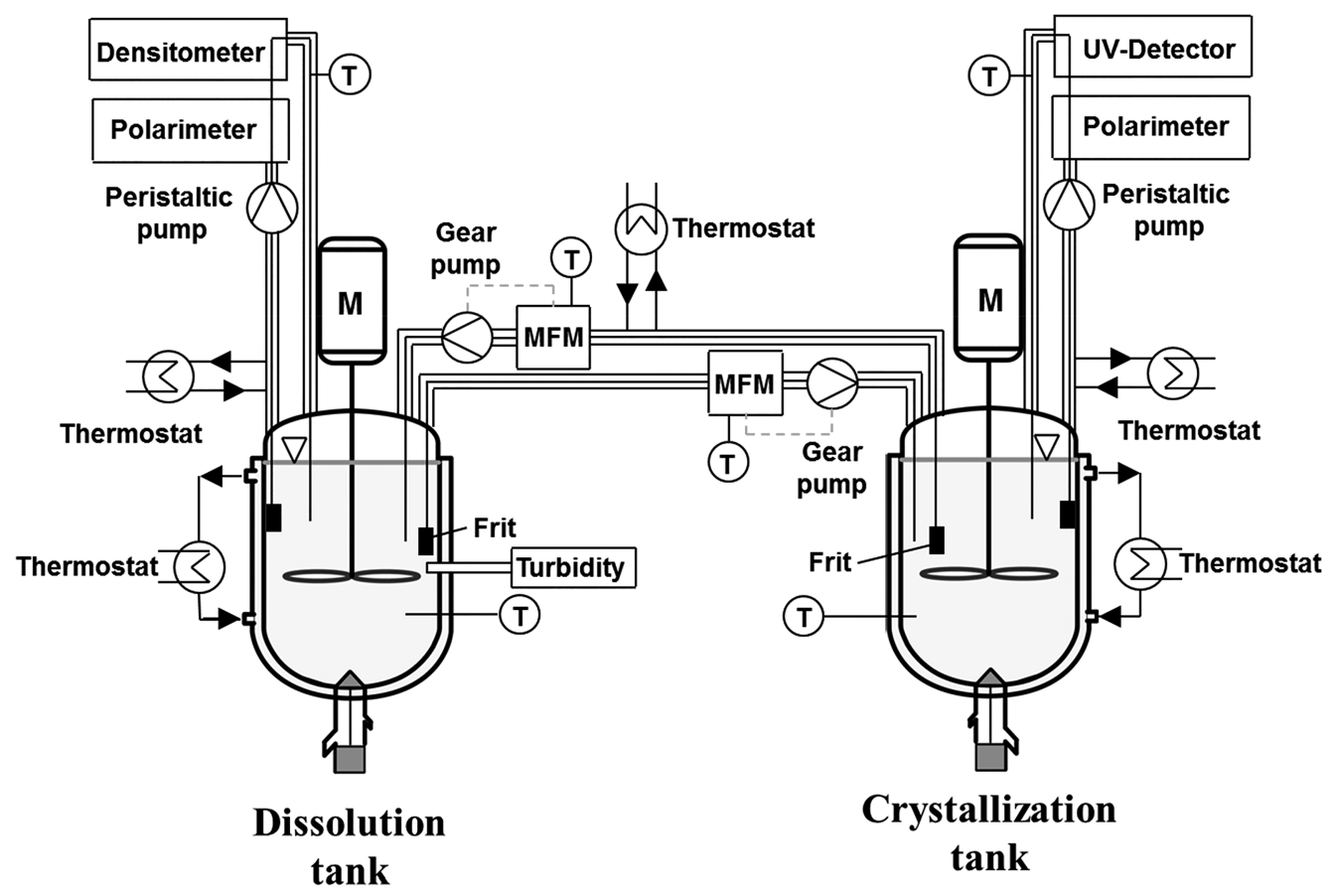

Figure 4. Coupled dissolution/crystallization plant consisting of two double-walled $450 \mathrm{~mL}$ tanks, two online analytic bypasses, and two liquid exchange sections. Frit - filter frit; M - stirrer motor; MFM - mass-flow meter; T - Pt100; Turb. - turbidity probe. 

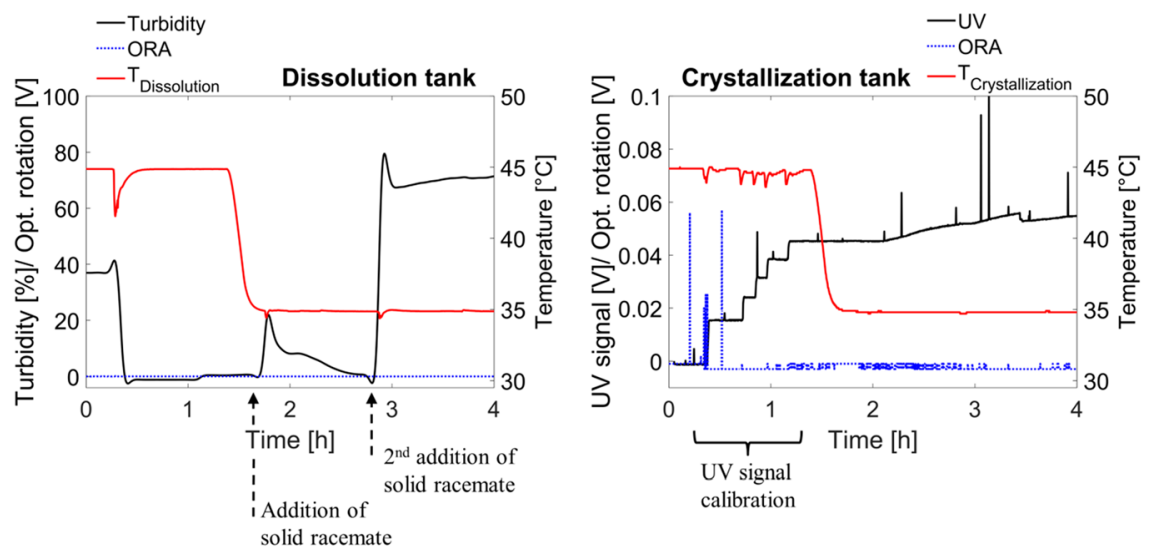

Figure 5. Startup-phase and UV calibration during the trial experiment $\left(T_{\text {sat }}=35^{\circ} \mathrm{C}, T_{\text {cryst }}=30-34{ }^{\circ} \mathrm{C}\right.$, Exp. 1 in Table 3$)$. Outliers or peaks in the UV and ORA signals in the crystallization tank originate from measured air bubbles, which is also valid for the following process trajectories shown (Figures 6, 9, and 10).
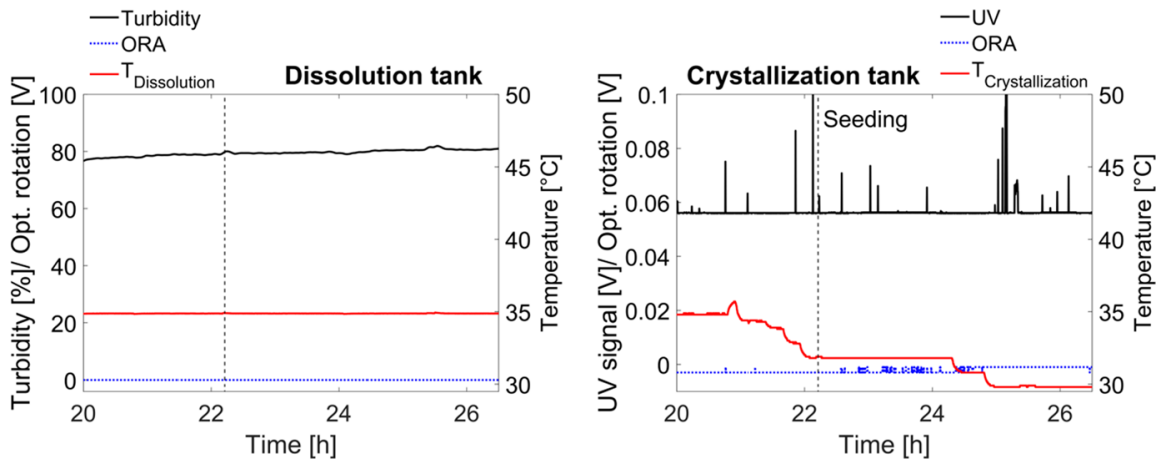

Figure 6. Dissolution and crystallization phase of the trial experiment after start-up and calibration shown in Figure $5\left(T_{\text {sat }}=35^{\circ} \mathrm{C}, T_{\text {cryst }}=30-34\right.$ ${ }^{\circ}$ C, Exp. 1 in Table 3).

blockage. The dissolution tank is additionally equipped with a turbidity probe (Mettler Toledo, Trb. 8300) to follow the decrease in suspension density. Pt100 sensors were used to measure the temperatures of each vessel and of the solution bypassed for online analyses.

2.4. HPLC Analysis. Samples of the crystalline products at the end of each experiment were investigated by HPLC with respect to purity and composition. A Chiralcel-OD-H $(4.6 \times 250 \mathrm{~mm}$, particle size 5 $\mu \mathrm{m})$ column was utilized with an Agilent 1200 system (Agilent Technologies) and 65/35 $n$-hexane/isopropanol as eluent. Flow rate, UV wavelength, column temperature, and injection volume were 1 $\mathrm{mL} / \mathrm{min}, 275 \mathrm{~nm}, 23{ }^{\circ} \mathrm{C}$, and $5 \mu \mathrm{L}$, respectively.

2.5. XRPD Analysis. The solid products were investigated with an X'Pert Pro Diffractometer (PANalytical $\mathrm{GmbH}$ ) using $\mathrm{Cu} \mathrm{K} \alpha$ radiation and an $\mathrm{X}^{\prime}$ Celerator detector in the $2 \theta$ range of $3-40^{\circ}$ with a step size of $0.017^{\circ}$ and a step time of 50 s per step.

No phase change was observed during the investigations, and always anhydrous guaifenesin was crystallized.

2.6. Experimental Procedure Development. The initial testing experiment is shown in Figures 5 and 6. A major challenge was to find suitable analytics to track the evolution of the liquid phase composition as well as the expected reduction in racemic suspension density in the dissolution tank caused by the selective removal of $(R)$ guaifenesin. In earlier studies on, e.g., threonine, ${ }^{7}$ a combination of density and optical rotation angle (ORA) measurement had proven as reliable techniques for process monitoring. However, it was not possible to follow the process trend by these measurement techniques alone due to the low specific ORA of guaifenesin and only slight liquid density changes during crystallization. A semipreparative UV detector was therefore additionally used in the crystallization tank, providing a better resolution of concentration changes.
CPCD is a quasi-steady-state process, and hence, no changes in the liquid phase composition are expected as long as process conditions are chosen appropriately. Nevertheless, the process needs to be interrupted when the provided solid material in the dissolution tank becomes enantiopure to prevent nucleation in the crystallization tank and to ensure maximal yields. The process equals afterward a preferential crystallization characterized by a decreasing concentration and no further removal of the initially racemic solid excess material in the dissolution tank. Hence, inline turbidity measurement was utilized in this tank to monitor and control the process. It is able to indicate the quantitative selective dissolution of the crystallizing enantiomer and the process end by reaching a lower plateau.

Figure 5 shows the preparation phase of the first experiment. On the basis of initial solubility data, ${ }^{10}$ solutions in both reactors were prepared to be saturated at $35^{\circ} \mathrm{C}$. The saturation point was chosen to avoid an eventual liquid-liquid demixing but enabling crystallization at moderate temperatures over a wide range of supersaturations (see Figure $3 \mathrm{~b}$ ). Five standards of racemic guaifenesin were added to the crystallization tank for calibration, which is indicated by the stepwise increase of the UV signal (Figure 5, right). Both tanks were kept at 45 ${ }^{\circ} \mathrm{C}$ during this period to ensure complete dissolution of the feed material.

After cooling of both tanks to saturation temperature, at $t \approx 1.7 \mathrm{~h}$, $20 \mathrm{~g}$ of solid racemate to be separated was added to the dissolution tank, resulting in a strong increase of the turbidity signal (Figure 5, left). Liquid exchange was subsequently turned on at a rate of $5 \mathrm{~mL} /$ min to equilibrate the solutions in both tanks. Unexpectedly, the added material dissolved quickly, which was detected by the decrease in turbidity (black line, dissolution tank, Figure 5, left) and the further increase of the UV signal at $t \approx 2 \mathrm{~h}$ (black line, crystallization tank, Figure 5, right). With a different process strategy (e.g., conventional 
PC or coupled $\mathrm{PC}^{13}$ ), the obvious inaccuracy of the solubility data would have been left undetected and a loss of the pure seed material would have been the result. An additional $50 \mathrm{~g}$ of racemate was added to the dissolution tank $(t \approx 2.8 \mathrm{~h})$, which was estimated to result in a suspension. The stabilization of the turbidity signal after $\approx 3.7 \mathrm{~h}$ indicated that no further dissolution took place. Nevertheless, both tanks were left overnight, exchanging mother liquor at a rate of $5 \mathrm{~mL} /$ $\min$ to ensure saturation of the liquid phases. Samples were withdrawn and analyzed gravimetrically to determine the actual liquid phase concentration before restarting the plant.

After subcooling the solution in the crystallization tank by $0.5 \mathrm{~K}$, $0.1 \mathrm{~g}$ of $(R)$-guaifenesin seeds was added to initiate the preferential crystallization process (black dashed line, crystallization tank, Figure 6, right).

Subsequently, temperature was decreased stepwise to identify suitable crystallization conditions. However, no significant changes in the signals were observed even at $30^{\circ} \mathrm{C}$ (supersaturation with respect to the racemic composition $\left.S_{\mathrm{rac}}=2.16\right)$. The suspension of the crystallization tank was finally harvested at $t=26.9 \mathrm{~h}$, filtered, and carefully washed with ice-cold water. Altogether, $0.8 \mathrm{~g}$ of $(R)$ guaifenesin was entrained with a purity of $98.52 \%$, proving the general feasibility of the process utilizing these conditions. However, the amount of solid racemic excess material in the dissolution tank was too high to be purified successfully. It was therefore reduced in subsequent experiments. Additionally, it was evident that guaifenesin growth kinetics are rather slow. In order to improve the overall process performance, the initial seed loading was also adapted to provide a larger crystallization surface area.

The experimental conditions were found applicable during the trial experiment (Table 2), which served as a starting point for further separation runs.

Table 2. General Process Parameters for the CPCD Separation Experiments

\begin{tabular}{lll}
\multicolumn{1}{c}{ parameter } & \multicolumn{1}{c}{ value } & unit \\
$T_{\text {sat }}$ & 35 & ${ }^{\circ} \mathrm{C}$ \\
$T_{\text {cryst }}$ & 30 & ${ }^{\circ} \mathrm{C}$ \\
$N_{\text {stirr }}$ & 200 & $\mathrm{rpm}$ \\
$m_{\mathrm{H}_{2} \mathrm{O}}$ & 400 & $\mathrm{~g}$ \\
$m_{\text {gua }}($ rac. $)$ & 53.3 & $\mathrm{~g}$ \\
$S_{\text {rac }}$ & 2.16 & - \\
$m_{\text {seed,R }}$ & $0.1-1.1$ & $\mathrm{~g}$ \\
$m_{\text {sol.rac.feed }}($ dissolution tank $)$ & $3.5-40$ & $\mathrm{~g}$ \\
$\dot{V}_{\text {exchange }}$ & $5-30$ & $\mathrm{~mL} / \mathrm{min}$ \\
\hline
\end{tabular}

\section{EXPERIMENTAL RESULTS}

3.1. Solubility and Metastable Zone Width. In the preliminary experiment, deviations from the polythermal solubility data obtained in ref 10 were observed. Hence, additional isothermal solubility measurements were carried out following the procedure described in section 2.2. Especially in the range of the steep increase of the saturation curve between 30 and $45{ }^{\circ} \mathrm{C}$, samples were prepared for temperature steps of $1 \mathrm{~K}$. Even though solid phases were dissolved before equilibration at a higher temperature, a solid phase recrystallized at the respective saturation temperature. Hence, no evidence was found supporting a stable liquid-liquid phase separation.

The measured saturation concentrations are depicted in Figure 7 (black crosses and dashed line), which show a similar trend as the data from ref 10 (black and red squares for polythermal and isothermal data, respectively). Although the few isothermal data points from ref 10 perfectly agree, the solubility curve is slightly shifted toward lower temperatures

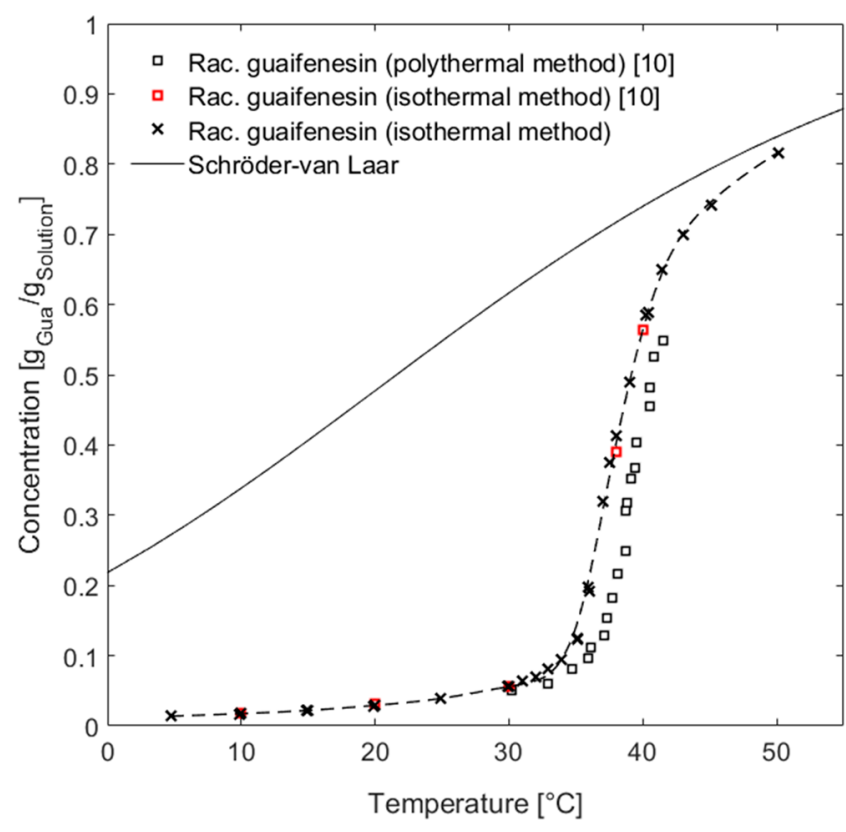

Figure 7. Measured solubility curve of racemic guaifenesin (isothermal method) in comparison with data from ref 10 and the ideal solubility line calculated according to the Schröder-van Laar equation. The dashed line is just a guide for the eye.

compared to results of polythermal studies reported in ref 10 , which explains the unexpected dissolution during the trial experiment. Particularly, in the range of the steepest slope of the solubility curve, deviations of around $20 \mathrm{wt} \%$ occur. The differences are probably caused by the very slow dissolution kinetics of guaifenesin, which influences the accuracy of the polythermal method, even though low heating ramps of 0.05 $\mathrm{K} /$ min were utilized in ref 10 . Also, no other than the known solid state form was found by XRPD analyses. The isothermal solubility results from all samples are listed in the Supporting Information (Table A1) together with the standard deviations of samples of one particular temperature.

Metastable zone widths were investigated for different concentrations with a polythermal method and are shown in Figure 8. As expected, the metastable zone width increases with increasing cooling rate. Further, it can be seen that, for high cooling rates $(-80$ and $-20 \mathrm{~K} / \mathrm{h})$, no correlation between nucleation points and the shape of the solubility curve is present. This can be explained by the crystallization of water and guaifenesin together, i.e., crystallization below the eutectic temperature, which is calculated to $-4{ }^{\circ} \mathrm{C}$ from the intersection of the liquidus lines according to the Schrödervan Laar equation. This explanation is supported by two different solid phases, which were observed inside the samples right after nucleation. For cooling rates between -0.5 and -5 $\mathrm{K} / \mathrm{h}$, the metastable zone widths, or the supersolubility curves, follow the trend of the solubility curve with possible subcoolings of the clear solutions ranging from 10 to $23 \mathrm{~K}$ $(-0.5 \mathrm{~K} / \mathrm{h})$ to $30-40 \mathrm{~K}(-5 \mathrm{~K} / \mathrm{h})$.

The recorded image data during nucleation revealed that crystals were formed directly from the clear solutions and no second liquid phase was observed. Thus, the hypothesis that a metastable miscibility gap is the reason for the unusual solubility behavior cannot be supported by this data.

3.2. Separation Results. The results of all separation experiments, including Exp. 1 described before, are compiled in 


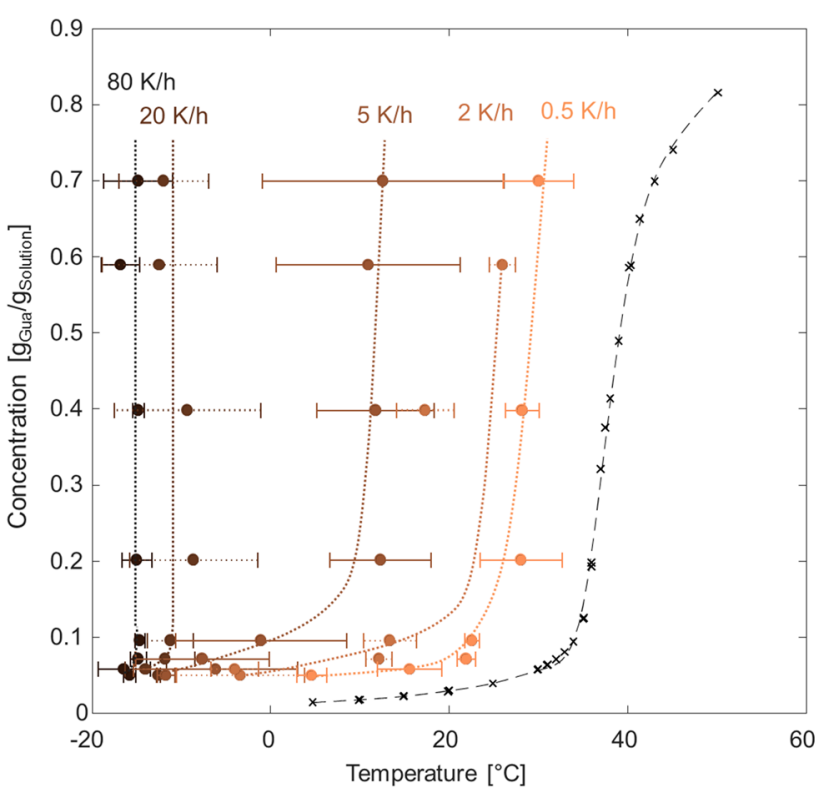

Figure 8. Identified nucleation points of solutions with different guaifenesin concentrations, which were linearly cooled at different rates $(0.5-80 \mathrm{~K} / \mathrm{h})$. Dashed and dotted lines are guides for the eye for the solubility curve and the supersolubility curves at various cooling rates, respectively.

Table 3. The essential initial parameters, the mass of seed material, $m_{\text {seed }}$ and solid racemic feed, $m_{\text {sol.feed, are listed }}$ together with the masses of produced solid phases in the crystallization and dissolution tank, $m_{\mathrm{R}}$ and $m_{\mathrm{S}}$. After HPLC analysis of the crystalline products, purity in both tanks, $\operatorname{Pur}_{R}$ and $\operatorname{Pur}_{S}$, was determined according to eq A1 (see Supporting Information). The listed productivities, $\operatorname{Pr}_{R}$ and $\operatorname{Pr}_{S}$, were calculated according to eq A2 (see Supporting Information). The process time, $t_{\text {proc }}$ was defined as the time between seeding the process and harvesting the product.

It can be seen from Table 3 that, in the first four experiments (Exps. 1-4), the mass of the solid racemic excess material, $m_{\text {sol.feed }}$ was adapted to achieve a complete separation and, hence, pure enantiomers in both tanks. With the process conditions listed in Table 2, $3.5 \mathrm{~g}$ of solid racemic feed was purified (Exp. 4 in Table 3) and $1.8 \mathrm{~g}$ of $(R)$ - and $1.2 \mathrm{~g}$ of $(S)$ guaifenesin with purities $>95 \%$ could be harvested. The initial seed, $m_{\text {seed, }}$ and racemic solid excess masses, $m_{\text {solfeed }}$ were increased, subsequent to this successful separation, to enhance yield and productivity of the process (Exps. 5-7). In the following experiments (Exps. 8-10), both enantiomers of guaifenesin were produced with high purities for further studies. In the last experiment (Exp. 11), the technical limit of the plant was investigated. An initial racemic solid excess mass was chosen, which allowed just for an appropriate liquid phase exchange with respect to filter blocking and exchange rate (technical limit $30 \mathrm{~mL} / \mathrm{min}$ ).

The online measurements of the first successful separation (Figure 9; Exp. 4, see Table 3) show similar trends compared to the trial experiment following the preparation procedure (dashed lines at $t=0 \mathrm{~h}$ in Figure 9). The stable UV signal indicates constant concentration in both tanks (black line, crystallization tank, Figure 9, right). The small increase of the optical rotation signal between $t=0-6 \mathrm{~h}$ (blue dotted line, crystallization tank, Figure 9, right) shows a slight enrichment of the unseeded enantiomer in the liquid phase. Hence, the chosen liquid phase exchange rate of $10 \mathrm{~mL} / \mathrm{min}$ was almost sufficient to counterbalance selective crystallization in the crystallization tank. After seeding (dashed line in Figure 9), the turbidity in the dissolution tank remains constant for almost 2 $\mathrm{h}$, indicating an initially slow preferential crystallization caused by the low seed loading. The signal starts to decrease significantly at $t \approx 2 \mathrm{~h}$ due to distinct selective dissolution in this tank and subsequently reaches a plateau $(t \approx 5 \mathrm{~h})$. Both enantiomers could have been harvested at this point already with high purity. However, to enhance productivity and yield, the temperature in the dissolution tank was lowered to the crystallization temperature as well, which transfers the CPCD process into a coupled preferential crystallization. ${ }^{13}$ Hence, both solid phases crystallize further and selectively remove the respective enantiomer from the liquid phase. This is indicated by a slight increase in turbidity showing that the process can in fact be monitored reliably with this method. Afterward, both products were withdrawn, filtered, and washed.

Seed mass and solid racemic excess material were increased stepwise for the following experiments to further enhance productivity. Figure 10 shows experiment 11 (compare Table

Table 3. Product Purities, $\operatorname{Pur}_{\mathrm{R}}$ and Pur $\mathrm{S}$, and Masses, $m_{\mathrm{R}}$ and $m_{\mathrm{S}}$ (Dry Product), as Well as Process Times, $t_{\text {proc }}$ and Productivities, $\operatorname{Pr}_{\mathrm{R}}$ and $\operatorname{Pr}_{\mathrm{S}}$, of All CPCD Experiments Performed ${ }^{a}$

\begin{tabular}{|c|c|c|c|c|c|c|c|c|c|}
\hline Exp. & $m_{\text {seed }}[\mathrm{g}]$ & $m_{\text {sol.feed }}[\mathrm{g}]$ & $m_{\mathrm{R}}[\mathrm{g}]$ & $\operatorname{Pur}_{R}[\%]$ & $m_{\mathrm{S}}[\mathrm{g}]$ & $\operatorname{Pur}_{S}[\%]$ & $t_{\text {proc }}[\mathrm{h}]$ & $\operatorname{Pr}_{R}[g /(\operatorname{kg~h})]$ & $\operatorname{Pr}_{S}[\mathrm{~g} /(\mathrm{kg} \mathrm{h})]$ \\
\hline 1 & 0.1 & (37) & 0.8 & 99 & & & 4.3 & 0.36 & \\
\hline 2 & 0.1 & 20 & 1.4 & 98 & & & 6.3 & 0.45 & \\
\hline 3 & 0.1 & 4 & 2.4 & 97 & 0.6 & 91 & 7.2 & 0.68 & 0.17 \\
\hline 4 & 0.1 & 3.5 & 1.8 & 98 & 1.2 & 95 & 6.7 & 0.55 & 0.37 \\
\hline 5 & 0.3 & 10 & 4.3 & 98 & 3.5 & 97 & 5.8 & 1.49 & 1.26 \\
\hline 6 & 1.1 & 20 & 19.6 & 80 & 11.2 & 98 & 3.5 & 9.31 & 6.63 \\
\hline 7 & 1.1 & 30 & 13.8 & 97 & 8.8 & 98 & 1.9 & 14.27 & 9.39 \\
\hline 8 & 1.1 & 30 & 31.6 & 69 & 8.9 & 98 & 3.3 & 14.03 & 5.47 \\
\hline 9 & 1.1 & 30 & 11.3 & 98 & 14.5 & 98 & 2.7 & 9.05 & 10.89 \\
\hline 10 & 1.1 & 30 & 16.1 & 93 & 14.9 & 98 & 4.0 & 7.67 & 7.55 \\
\hline 11 & 1.1 & 40 & 32.7 & 97 & 16.9 & 98 & 4.0 & 16.86 & 9.13 \\
\hline
\end{tabular}

${ }^{a}$ Seeding was always done with $(R)$-guaifenesin except for Exp. 9, where the $(S)$-enantiomer was used. 

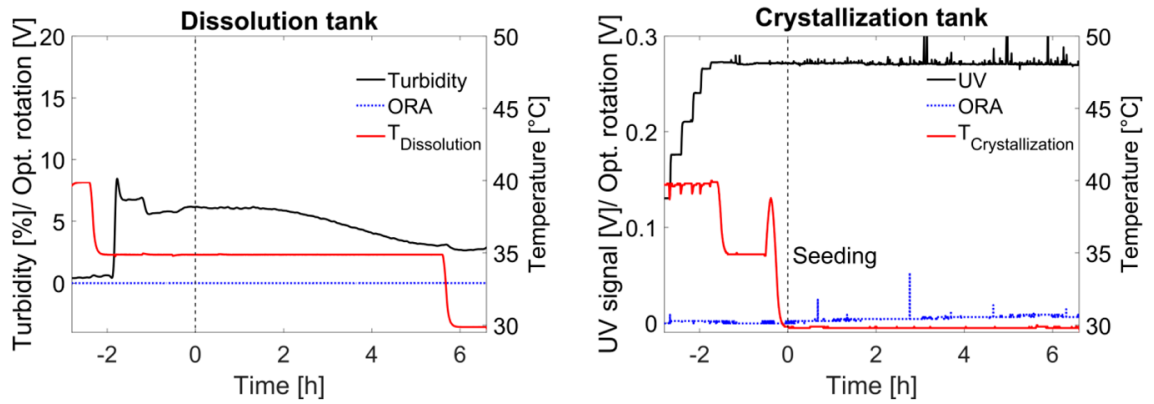

Figure 9. Trajectories of turbidity, optical rotation (optical rotation angle, ORA), UV absorption, and temperature of CPCD experiment $4\left(T_{\text {sat }}=\right.$ $35^{\circ} \mathrm{C}, \mathrm{T}_{\text {cryst }}=30^{\circ} \mathrm{C}$, Exp. 4 in Table 3 ).
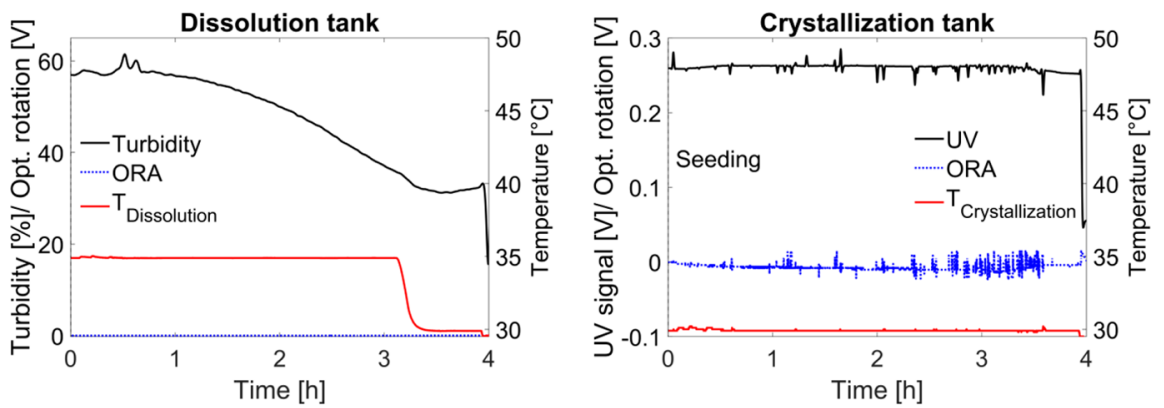

Figure 10. Trajectories of turbidity, optical rotation (optical rotation angle, ORA), UV absorption, and temperature of CPCD experiment 11 ( $T_{\text {sat }}$ $=35^{\circ} \mathrm{C}, T_{\text {cryst }}=30^{\circ} \mathrm{C}$, Exp. 11 in Table 3 ).

3) where $40 \mathrm{~g}$ of racemic guaifenesin was separated. The larger mass of carefully ground seeds decreased the initial startup period to $1 \mathrm{~h}$ and the overall process time until a complete purification was achieved after $4 \mathrm{~h}$. A liquid exchange rate of 30 $\mathrm{mL} / \mathrm{min}$ between both tanks was necessary to keep the mother liquor at racemic composition in this case. A slight decrease of the UV signal can be seen in the last $0.5 \mathrm{~h}$ (black line in Figure 10, crystallization tank, right) due to the preferential crystallization of both enantiomers from the liquid phase at the end of the experiment, which fits well with the final increase in turbidity in the dissolution tank. In total, $33 \mathrm{~g}$ of $(R)$ - and $17 \mathrm{~g}$ of $(S)$-guaifenesin were produced with purities > 97\%. The high productivities, which were achieved $\left(\operatorname{Pr}_{\mathrm{R}} \approx 17\right.$ $\left.\mathrm{g} /(\mathrm{kg} \cdot \mathrm{h}) ; \operatorname{Pr}_{\mathrm{S}} \approx 9 \mathrm{~g} /(\mathrm{kg} \cdot \mathrm{h})\right)$, show the great potential of the CPCD process also for industrial applications.

The empirical process control strategy based on the utilized measurement techniques can be summarized as follows. If the turbidity signal reaches a lower plateau and all other signals are almost constant, the process can be interrupted since the seeded enantiomer is completely removed from the solid excess material in the dissolution tank. An accelerating decrease of this signal without any plateau indicates primary nucleation of the unseeded enantiomer in the crystallization tank, followed by complete loss of the solid phase in the dissolution stage. In this case, the seed mass or the driving force needs to be lowered to reduce the rate of selective entrainment in the crystallization tank. In the opposite case, if the turbidity signal stays constant, supersaturation and seed loading have to be increased to enhance process performance. A change of the optical rotation or the UV absorption in the crystallization stage indicates the selective depletion of the seeded enantiomer from the mother liquor, increasing the risk of primary nucleation of the antipode. The liquid exchange rate between both tanks needs to be increased in this case, to counterbalance the entrainment by selective dissolution.

It can be seen from the purities in Table 3 that primary nucleation of the counter-enantiomer cannot always be prevented in the crystallization tank. If the process is optimized and higher supersaturations are utilized, the probability of nucleation of the antipode increases rapidly. This is caused by the inherently given nonrobustness of the preferential crystallization process. A successful separation is still possible even if nucleation of the unseeded enantiomer occurs in the crystallization tank. In this case, the crystallization tank should become the dissolution tank and vice versa until the nucleated crystals are dissolved again. However, this requires a precise analysis of the solid phase composition in each tank.

Nevertheless, below a certain supersaturation, no nucleation will occur in a finite time but at the expense of process performance with respect to yield and productivity. In this case, a change of the setup could be beneficial if, for example, a wet-milling process is introduced to generate more crystal surface and, hence, increase the productivity even at low supersaturations.

By contrast, primary nucleation is completely avoided in the dissolution tank; thus pure product will always be gained in this vessel if crystallization is carried out for a sufficiently long duration.

Only a part of the product here can be lost if the unseeded enantiomer nucleates in the crystallization tank. Hence, the process needs to be adapted to every substance system, specifically utilizing appropriate models or control strategies like the aforementioned empirical control to achieve the highest productivities and still meet the purity constraints in both vessels. 


\section{CONCLUSIONS}

The study has shown that the presented CPCD process is capable of efficiently resolving racemic guaifenesin, providing both enantiomers simultaneously in a highly pure crystalline form. A sufficient amount of pure material was produced in two connected lab-scale tanks for further studies, starting from an initially small quantity of seeds even though slow growth kinetics complicated the separations and deviations to the reported solubility curve were found. This demonstrates the advantages of the applied quasi-stationary process. Crystallization conditions can be chosen in a way that no or negligible nucleation of the unseeded enantiomer occurs to keep the process metastable until a significant entrainment of the preferred enantiomer is visible. An equilibration step during the preparation procedure of the process decreases the risk of unexpected dissolution of expensive enantiopure seed material because of the presence of excess solid racemic material. Problems arising from inaccurate solubility data or maybe also as a result of different batches of starting racemate can thus be mitigated.

The solubility of racemic guaifenesin was determined with an isothermal method while metastable zone width measurements were carried out using polythermal conditions. The trend of the reported solubility data was verified, but a small shift with respect to temperature was found. It was shown that guaifenesin/water solutions have a rather large zone of metastability, which increases strongly with faster cooling rates. Neither a stable nor metastable liquid-liquid demixing was found during these investigations.

The process was designed rationally on the basis of a few trial crystallizations, which yielded appropriate operating conditions for a complete separation. Subsequently, the operating conditions were adapted, mainly seed loading and preparation as well as the mass of solid racemic excess material, to increase yield and productivity. In a final experiment, a maximal productivity of $16.9 \mathrm{~g} /(\mathrm{kg} \cdot \mathrm{h})$ in the crystallization and $9.1 \mathrm{~g} /(\mathrm{kg} \cdot \mathrm{h})$ in the dissolution tanks were achieved with purities exceeding $97 \%$. From all observations of the performed experiments, maximal productivities, which are mainly based on an inline turbidity probe, were derived to enhance the applicability of this process to other systems.

All separation experiments combined in this study yielded $136 \mathrm{~g}$ of the $(R)$-guaifenesin product and $81 \mathrm{~g}$ of the $(S)$ guaifenesin product with average purities of $88 \%$ and $98 \%$, respectively, starting from only $0.8 \mathrm{~g}$ of pure $(R)$-guaifenesin. These values were improved to $>99.9 \%$ by simple recrystallization. The lower purity of the $(R)$-enantiomer results obviously from primary nucleation of the counter-enantiomer, showing potential for further improvement of the current process.

\section{ASSOCIATED CONTENT}

\section{S Supporting Information}

The Supporting Information is available free of charge on the ACS Publications website at DOI: 10.1021/acs.cgd.8b01660.

Equations utilized for the calculation of purity and productivity, and table of isothermal solubility data (PDF)

\section{AUTHOR INFORMATION}

\section{Corresponding Author}

*E-mail: lorenz@mpi-magdeburg.mpg.de. Tel: +49391 6110293. Fax: +493916110500.
ORCID

Erik Temmel: 0000-0001-5822-5817

Heike Lorenz: 0000-0001-7608-0092

Notes

The authors declare no competing financial interest.

\section{ACKNOWLEDGMENTS}

The cooperation with the Arbuzov Institute of Organic and Physical Chemistry (Russian Academy of Sciences, Kazan) is gratefully acknowledged. The authors thank especially Prof. Alexander A. Bredikhin and Dr. Robert Fayzullin for providing $\approx 1 \mathrm{~g}$ of pure $(R)$-guaifenesin. This research received funding as part of a CORE project (October 2016-September 2020) from the Horizon2020 Research and Innovation Programme of the European Union under Marie Sklodowska-Curie Grant Agreement No. 722456 CORE ITN.

\section{NOMENCLATURE}

\section{Symbols}

M [-]=Motor

$m[\mathrm{~g}]=$ Mass

$n[\mathrm{rpm}]=$ Stirrer speed

ORA $[\mathrm{V}]=$ Signal of the optical rotation angle

$\operatorname{Pr}[\mathrm{g} /(\mathrm{kg} \cdot \mathrm{h})]=$ Productivity

Pur $[\%]=$ Purity

$S[-]=$ Relative supersaturation

$T\left[{ }^{\circ} \mathrm{C}\right]=$ Temperature

Turb [-]=Turbidity

$t[\mathrm{~h}]=$ Time

$x[-]=$ Mass fraction

$\mathrm{UV}[\mathrm{V}]=$ Signal of the UV detector

$\dot{V}[\mathrm{~mL} / \mathrm{min}]=$ Volumetric flow rate

\section{Subscripts and Superscripts}

cryst $=$ Crystallization

feed $=$ Feed

gua $=$ Guaifenesin

$\mathrm{H}_{2} \mathrm{O}=$ Water

$i=$ Component $i$

proc $=$ Process

rac $=$ Racemic

sat $=$ Saturation

stirr=Stirrer

seed $=$ Seed

sol.=Solid

solution=Solution

$0=$ Initial value

$R / S=(R)-/(S)$-enantiomer of guaifenesin

\section{REFERENCES}

(1) Lin, G.-Q., You, Q.-D., Cheng, J.-F., Eds. Chiral Drugs: Chemistry and Biological Action; John Wiley \& Sons, Inc.: Hoboken, NJ, 2011.

(2) Garrison, A. W., Gan, J., Liu, W., Eds. Chiral Pesticides: Stereoselectivity and Its Consequences; American Chemical Society: Washington, DC, 2012.

(3) Lorenz, H.; Seidel-Morgenstern, A. Processes To Separate Enantiomers. Angew. Chem., Int. Ed. 2014, 53, 1218-1250.

(4) Coquerel, G. Preferential Crystallization. In Novel Optical Resolution Technologies; Springer: Berlin, 2006; pp 19-41.

(5) Lorenz, H.; Perlberg, A.; Sapoundjiev, D.; Elsner, M. P.; SeidelMorgenstern, A. Crystallization of enantiomers. Chem. Eng. Process. 2006, 45, 863-873.

(6) Bredikhina, Z. A.; Novikova, V. G.; Zakharychev, D. V.; Bredikhin, A. A. Solid state properties and effective resolution 
procedure for guaifenesin, 3-(2-methoxyphenoxy)-1,2-propanediol. Tetrahedron: Asymmetry 2006, 17, 3015-3020.

(7) Levilain, G.; Eicke, M. J.; Seidel-Morgenstern, A. Efficient Resolution of Enantiomers by Coupling Preferential Crystallization and Dissolution. Part 1: Experimental Proof of Principle. Cryst. Growth Des. 2012, 12, 5396-5401.

(8) Eicke, M. J.; Levilain, G.; Seidel-Morgenstern, A. Efficient Resolution of Enantiomers by Coupling Preferential Crystallization and Dissolution. Part 2. A Parametric Simulation Study To Identify Suitable Process Conditions. Cryst. Growth Des. 2013, 13, 16381648.

(9) Bredikhin, A. A.; Bredikhina, Z. A.; Zakharychev, D. V.; Pashagin, A. V. Pashagin, Chiral drugs related to guaifenesin: synthesis and phase properties of methocarbamol and mephenoxalone. Tetrahedron: Asymmetry 2007, 18, 1239-1244.

(10) Fayzullin, R. R.; Lorenz, H.; Bredikhina, Z. A.; Bredikhin, A. A.; Seidel-Morgenstern, A. Solubility and Some Crystallization Properties of Conglomerate Forming Chiral Drug Guaifenesin in Water. J. Pharm. Sci. 2014, 103, 3176-3182.

(11) Kaduk, J. A. Crystal structure of guaifenesin, 3-(2-methoxyphenoxy)-1,2-propanediol. Powder Diffr. 2004, 19, 127-132.

(12) Material Safety Data Sheet. Guaiacol Glycerol Ether G0159; TCI Germany GmbH: Frankfurt, 2018.

(13) Elsner, M. P.; Ziomek, G.; Seidel-Morgenstern, A. Simultaneous preferential crystallization in a coupled batch operation mode. Part II: Experimental study and model refinement. Chem. Eng. Sci. 2011, 66, 1269-1284. 